
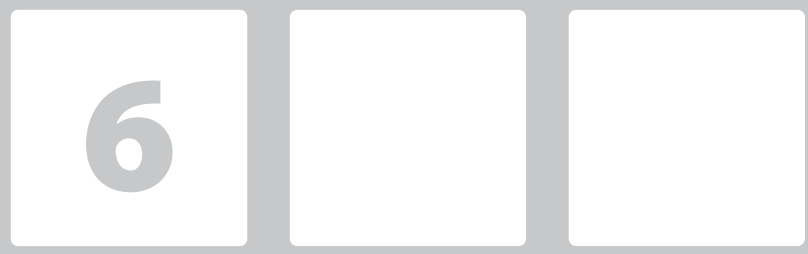

\title{
ANÁLISE DE EFICIÊNCIA DAS IFES NO USO DE RECURSOS FINANCEIROS: UMA APLICAÇÃO DEA EM DOIS ESTÁGIOS
}

\section{Analysis of the efficiency of IFES in the use of financial resources: a DEA application in two stages}

\author{
Antonio Marcos Correia Melonio \\ Universidade de Brasília - UnB. \\ email:amelonio@unb.br
}

\author{
Vander Mendes Lucas \\ Universidade de Brasília - UnB. \\ email:vlucas@unb.br
}

\section{RESUMO}

As Instituições Federais de Ensino Superior (IFES) são compostas principalmente por universidades, sendo que desempenham um papel estratégico no desenvolvimento econômico, social, científico e tecnológico do país. Este estudo buscou avaliar a eficiência das IFES em relação aos gastos de recursos públicos do programa Reestruturação e Expansão das Universidades Federais (REUNI) em 2015 e verificar a relação de eficiência das IFES com variáveis ambientais. A metodologia empregada para medir essa eficiência das IFES foi a Análise Envoltória de Dados (DEA) em dois estágios e a Regressão Tobit. O cálculo da análise de eficiência para o ano 2015 mostrou que 24 IFES foram consideradas eficientes, correspondendo a $44 \%$ da amostra, sendo que se destacaram dentre as eficientes a Universidade Federal do Estado do Rio de Janeiro, a Universidade Federal de Minas Gerais, a Universidade Federal dos Vales do Jequitinhonha e Mucuri, a Universidade Federal do Amapá, a Universidade Federal do Pará e a Universidade Federal do Rio de Janeiro. Na análise de Regressão Tobit verificou-se a adequação do modelo de eficiência das IFES e como essa eficiência está relacionada com as variáveis ambientais.

Palavras-Chave: DEA em dois estágios; IFES; Regressão Tobit; REUNI

\section{ABSTRACT}

The Federal Higher Education Institutions (FHEI) are mainly composed of universities and they play a strategic role in the country's economic, social, scientific and technological development. This study sought to evaluate the efficiency of FHEl in relation to the expenditure of public resources of the Federal Universities Restructuring and Expansion (FURE) program in 2015 and to verify the efficiency ratio of FHEl with environmental variables. The methodology used to measure this efficiency of the FHEl was Data Envelopment Analysis (DEA) in two stages and the Tobit Regression. The calculation of the efficiency analysis for the year 2015 showed that 24 FHEI were considered efficient, corresponding to $44 \%$ of the sample, of which Federal University of the State of Rio de Janeiro, Federal University of Minas Gerais, Federal University of Vales do Jequitinhonha and Mucuri, Federal University of Amapá, Federal University of Pará and Federal University of Rio de Janeiro stood out among the efficient ones. In the Tobit Regression analysis the adequacy of the FHEl efficiency model was verified and how this efficiency is related to the environmental variables.

Key-words: DEA in two stages; FHEl; Tobit Regression; FURE 


\section{INTRODUÇÃO}

No Brasil, assim como no mundo, a segunda metade do século XX foi marcada por uma grande expansão da demanda e da oferta de cursos de educação superior, relacionadas tanto ao conhecimento acadêmico quanto ao crescimento da pesquisa. Nos países em desenvolvimento a educação possui particularidades, decorrentes da desigualdade regional, da má distribuição de renda, baixa escolaridade, recursos limitados e dificuldades de acesso e permanência no ensino superior. No contexto brasileiro, mais especificamente, os desafios da educação superior podem ser concentrados no triple expansão, qualidade e democratização do ensino. No período de 2003 a 2015, o Ministério de Educação (MEC) se pautou em uma série de medidas e conceitos, dentre as quais está o resultado de 9.306.877 de pessoas que concluíram o curso de ensino superior no período, o que corresponde a 5\% da população brasileira, segundo o Censo do 2010 (BRASIL, 2016).

A educação de ensino superior é de fundamental importância estratégica para o desenvolvimento social e econômico de qualquer país. De acordo com Menezes e Santos (2002), as Instituições Federais de Ensino Superior (IFES) são um conjunto de instituições criadas ou incorporadas e mantidas pelo governo, constituindo o Sistema de Instituições Federais de Ensino Superior e a Rede Pública de Ensino. As IFES também são compostas por universidades, instituições isoladas e pelos centros de ensino tecnológico, sendo que desempenham papel relevante no desenvolvimento científico e tecnológico do país, respondendo, aproximadamente, por $90 \%$ da produção científica e pela formação de diversos profissionais.

O governo federal tem buscado, com várias medidas, o crescimento do ensino superior público criando condições para que as universidades federais promovam a expansão física, acadêmica e pedagógica. Para tanto, o governo busca a expansão da educação superior, por meio de várias ações (SAVIANI, 2007), dentre elas está o Programa de Apoio a Planos de Reestruturação e Expansão das Universidades Federais (REUNI), que tem como principais objetivos ampliar o acesso e a permanência na educação supe- rior. As ações contidas no programa visam o aumento de vagas nos cursos de graduação, a ampliação da oferta de cursos noturnos, a promoção de inovações pedagógicas e o combate à evasão, com a finalidade, dentre outras, de diminuir as desigualdades sociais no país e promover o desenvolvimento econômico (BRASIL, 2007). O REUNI é uma das ações que integram o Plano de Desenvolvimento da Educação (BRASIL, 2001) e foi instituído pelo Decreto $n^{\circ}$ 6.096, de 24 de abril de 2007.

Os programas de governo voltados para o ensino superior público demandam uma quantidade significativa de recursos financeiros, para que essas instituições possam desenvolver suas atividades com qualidade. Entretanto, a gestão desses recursos deve ser realizada com base nos princípios que regem a administração pública, dos quais destacamos a eficiência (BRASIL, 1988). A eficiência está relacionada à maneira como um sistema utiliza os recursos disponíveis, a fim de otimizar seus resultados. É um critério econômico que mostra a capacidade administrativa de realizar o máximo com uma certa quantidade de recursos.

As IFES estão cada vez mais pressionadas a prestarem contas à sociedade e aos órgãos reguladores e fiscalizadores do país, acerca dos impactos e resultados de suas ações, uma vez que os seus recursos financeiros são verbas públicas provenientes em geral de impostos (CAVALCANTE; ANDRIOLA, 2012). E devido à escassez de recursos financeiros e às disputas envolvidas no momento da distribuição, a questão é se as IFES são eficientes em relação ao custo-benefício dos recursos públicos aplicados (SILVA et al. 2007). Este artigo busca verificar a eficiência das IFES no ano de 2015, pois são utilizados nessas instituições recursos públicos financeiros do programa REUNI, assim como observar a validação dessa eficiência.

\section{REFERENCIAL TEÓRICO}

Este tópico trata do embasamento empírico e teórico com base na literatura sobre o Programa REUNI, da seleção das variáveis, dos atributos principais da eficiência e sua mensuração, e da regressão Tobit. 


\subsection{O Programa REUNI}

O Governo Federal tem lançado uma série de programas que visa realizar a expansão do ensino superior no país, não só quantitativa como também qualitativamente, tais como: o Programa de Apoio ao Plano de Reestruturação e Expansão das Universidades Federais (REUNI) para realizar a expansão de novos campi nas universidades públicas existentes; $\mathrm{o}$ Programa Universidade para Todos (PROUNI) que está sendo ampliado e agora atuando juntamente ao Financiamento ao Estudante do Ensino Superior (FIES); a criação de novos Institutos Federais de Educação, Ciência e Tecnologia (IFETs); e também a Universidade Aberta do Brasil - UAB que objetiva o aumento da educação superior à distância, criando vários polos em todo país, inclusive em regiões até então não privilegiadas com a educação superior (COSTA; PAIVA; FERREIRA, 2010). O REUNI que foi instituído pelo Decreto n. ${ }^{\circ} 6.096$ de 24 de abril de 2007, que, além de buscar atender a diversas reivindicações e a metas estabelecidas no Plano Nacional de Educação (BRASIL, 2001), buscou a expansão e reordenamento da educação superior federal no Brasil.

Em seu artigo primeiro, este decreto define o objetivo e a metas globais do REUNI, ficando instituído o Programa de Apoio a Planos de Reestruturação e Expansão das Universidades Federais (REUNI), com o objetivo de criar condições para a ampliação do acesso e permanência na educação superior, em nível de graduação, para melhor aproveitamento da estrutura física e de recursos humanos existentes nas universidades federais. No seu primeiro parágrafo, o Programa apresenta como meta global a elevação gradual da taxa de conclusão média dos cursos de graduação presenciais para $90 \%$ e da relação de 18 alunos de graduação em cursos presenciais por professor, no final de cinco anos, a contar do início de cada plano (BRASIL, 2007).

O REUNI fazia parte de uma política nacional de consolidação e expansão da educação superior federal, sendo que o Plano Nacional de Educação (PNE) previa o provimento da oferta de educação superior para, pelo menos, $30 \%$ dos jovens na faixa etária de 18 a 24 anos, até o final de 2010 (BRASIL, 2001). O programa REUNI fundamentava-se como uma chamada pública, em que não havia uma concorrência para seleção e apoio aos Planos de Reestruturação e Expansão, que foram aprovados previamente pelos conselhos superiores das universidades federais. Não era obrigatório a adesão ao Programa, porém 53 universidades federais aderiram ao REUNI, o que significava na época a sua totalidade (SILVA; FREITAS; LINS, 2013).

\subsection{A Seleção das Variáveis}

Apesar de algumas universidades conterem nos seus Relatórios de Gestão as informações adicionais referentes à qualidade dos serviços prestados pelas mesmas e outros indicadores que poderiam ser utilizados para melhor medir sua eficiência, não existe uma padronização dessas informações, por isso não encontraríamos as mesmas variáveis em todas as IFES. Os indicadores do Tribunal de Contas da União - TCU (BRASIL, 2002), por serem demandados pela legislação, conseguem propor um padrão na mensuração, o que permite avaliar os mesmos aspectos das variáveis em todas as IFES, por isso foram os escolhidos para o presente estudo.

Os indicadores do TCU têm como objetivo estimar aspectos do desempenho das instituições de ensino superior (BARBOSA; FREIRE; CRISÓSTOMO, 2011), e vem sendo utilizados em trabalhos recentes sobre a eficiência das IFES, tais como: Costa et. al. (2012), Oliveira (2013) e Siqueira (2015). Os indicadores que contam com o hospital universitário não foram considerados, haja vista que nem todas as universidades possuem tal instituição.

Segue abaixo a relação dos nove indicadores:

4. O indicador Custo Corrente/Aluno Equivalente (sem hospital universitário): representa a relação entre as despesas correntes de todas as unidades gestoras (menos as despesas com sentenças judiciais, aposentadorias, reformas e pensões, pessoal afastado ou cedido e, também, 100\% das despesas correntes dos hospitais universitários e maternidade) e o número de aluno equivalente, que é o número total de alunos da graduação, mais o número de alunos em tempo integral de pós-graduação e de residência médica.

5. O indicador Aluno Tempo Integral / Professor Equivalente: evidencia a relação entre o número 
de alunos em tempo integral e o número de professores equivalentes. Para encontrar o número de professor equivalentes há os seguintes pesos: 0,5 para 20 horas por semana e 1 para dedicação exclusiva ou 40 horas por semana. Professores afastados para capacitação e mandato eletivo ou cedidos para outros órgãos e/ou entidades da administração pública, em 31/12 do exercício, não integram o cálculo do indicador. Ou seja, esse indicador representa proporcionalmente a quantidade de alunos que está sob a tutela acadêmica de um professor.

6. O indicador Aluno Tempo Integral / Funcionário Equivalente (sem hospital universitário): representa a relação de alunos em tempo integral pelo número de funcionários equivalentes, que é obtido por meio dos seguintes pesos: 0,5 para 20 horas por semana, 0,75 para 30 horas por semana e 1 para 40 horas por semana. Esse indicador busca demonstrar a produtividade e a eficiência dos funcionários de uma instituição, a partir do cálculo do número médio de alunos por funcionário.

7. O indicador Funcionário Equivalente/Professor (sem hospital universitário): representa a relação entre o número de funcionários equivalentes sem HU e o número de professores equivalentes, cujas variáveis já foram explicitadas nos indicadores anteriores.

8. O indicador Grau de Participação Estudantil (GPE): é obtido por meio da razão entre o número de alunos com dedicação em tempo integral e o número total de alunos matriculados nos cursos de graduação. Este índice leva em consideração o número de diplomados, no ano letivo referente ao exercício, em cada curso; a duração padrão do curso, de acordo com a tabela da SESu; e o número de alunos que ingressaram, no ano letivo relativo ao exercício, em cada curso segundo fator de retenção calculado de acordo com metodologia da SESu. Dessa forma, ele expressa o grau de utilização, pelo corpo discente, da capacidade instalada das IFES e a velocidade de integralização curricular.

9. O indicador Grau de Envolvimento Discente com Pós-Graduação (GEPG): é alcançado por meio da divisão do total de alunos de pós-gra- duação (stricto sensu) pela soma do total de alunos de graduação e pós-graduação.

10. O indicador Conceito CAPES/MEC: faz referência à qualidade dos cursos de pós-graduação stricto sensu, sendo obtido pela média aritmética das notas de avaliação realizada pela Coordenação de Aperfeiçoamento de Pessoal de Nível Superior (CAPES) de todos os cursos de mestrado e doutorado (não considerando os mestrados profissionalizantes) e o número de programas de pós-graduação.

11. O indicador Índice de Qualificação do Corpo Docente (IQCD): é obtido, num primeiro momento, atribuindo-se peso 5 para doutores, peso 3 para mestres, peso 2 para docentes com especialização e peso 1 para os graduados. Depois, divide-se o resultado obtido pelo número total de professores. Sendo o índice máximo alcançável de 5 .

12. O indicador Taxa de Sucesso na Graduação (TSG): representa a relação entre o número de diplomados e o número total de alunos ingressantes. Devendo-se considerar o número de concluintes (que completaram os créditos, mesmo não tendo colado grau) dos cursos no ano letivo correspondente ao exercício. Esse indicador apresenta de forma inversa o grau de evasão dos alunos que ingressam nas IFES.

As variáveis apresentadas mostram por um lado medidas de qualidade e quantidade, de recursos consumidos pelas IFES e, por outro lado, as realizações a partir da utilização desses recursos, o que permite efetuar o cálculo da eficiência.

\subsection{A Eficiência Relativa}

A Eficiência está relacionada à maneira pela qual um sistema utiliza os recursos disponíveis, com objetivo de otimizar seus resultados. É um critério econômico que mostra a capacidade administrativa de produzir o máximo de resultados com o mínimo de recursos possíveis (BELLONI, 2000).

O cálculo da Eficiência é abordado com base na eficiência relativa, ou seja, a eficiência de Unidades Tomadoras de Decisão (traduzido do inglês Decision Making Units - DMUs) em relação a outras Unidades 
Tomadoras de Decisão (DMUs) que utilizam os mesmos inputs e outputs e praticam tarefas semelhantes (RIOS, 2005). A eficiência relativa de cada DMU é definida como a razão da soma ponderada de seus produtos (outputs) pela soma ponderada dos insumos necessários para gerá-los (inputs).

Os modelos de Análise Envoltória de Dados, neste artigo usaremos a sigla DEA (Data Envelopment Analysis) a qual é mais conhecida em inglês para referenciar esses modelos, têm sido aplicados com sucesso no estudo da eficiência. Essa técnica permite que nos estudos na área de educação, seja possível classificar as IFES mais eficientes quanto à alocação de recursos financeiros, baseado nos insumos utilizados e nos resultados produzidos (PEÑA, 2008).

A Análise Envoltória de Dados, desenvolvida por Charles, Cooper e Rhodes em 1978, é um método não paramétrico, que usa basicamente uma técnica de programação linear para calcular a eficiência comparada de unidades de produção, chamadas de Unidades Tomadoras de Decisão (DMUs), comparando os recursos (inputs) utilizados e os resultados (outpus) obtidos em cada DMUs, com todas as outras restantes. A DEA além de identificar as unidades mais eficientes em uma população, também fornece a medida da ineficiência para todas as outras. Cabe ressaltar que a DEA não mede a eficiência absoluta, e sim a eficiência relativa (RIOS, 2005). A DEA utiliza múltiplas variáveis que são classificadas em insumos que são chamados de "input" e produtos que são chamados de "output". Os modelos DEA trazem outra definição com relação à orientação, que podem ser orientados por input, por output, ou por ambos. A orientação por input minimiza o suficiente (insumos) para se alcançar um nível de produção desejado. A orientação por output significa maximizar os produtos (output) obtidos, mantendo constantes os recursos definidos como inputs. A orientação para ambos significa a busca pela máxima eficiência, minimizando os inputs e maximizando os outputs (RIOS, 2005).

A Eficiência Técnica é um conceito relativo que compara o que foi produzido por unidade de insumo utilizado com o que poderia ser produzido, do seguinte modo: (Produto / Insumo) realizada, comparada com (Produto / Insumo) mais adequada (FERREIRA; GOMES, 2009). Assim, a definição geral de eficiência técnica de uma organização ou atividade produtiva, quando se comparam duas ou mais dessas organizações, está relacionada à produção de um bem ou serviço com a menor utilização possível de recursos, ou seja, eliminando-se as folgas. Por sua vez, a forma de utilização dos recursos necessários para a produção está relacionada à tecnologia adotada e ao respectivo processo de produção, ou seja, modos de combinação de insumos de cada tecnologia (FERREIRA; GOMES, 2009).

No início da Análise Envoltória de Dados, o modelo proposto por Charnes, Cooper e Rhodes (1978), chamado de CCR por causa das iniciais dos nomes dos autores, foi desenhado para uma análise com retornos constantes de escala (CRS - Constant Returns to Scale), ou seja, qualquer variação nos insumos (inputs) produz variações proporcionais nos produtos (outputs).

A formulação para um problema de otimização, para o modelo DEA com retornos constantes de escala, pode ser visto na seguinte expressão:

$\max E f f_{0}=\left(\frac{\sum_{j=1}^{s} u_{j} y_{j 0}}{\sum_{i=1}^{r} v_{i} x_{i 0}}\right)$
sujeito a $\frac{\sum_{j=1}^{s} u_{j} y_{j k}}{\sum_{i=1}^{r} v_{i} x_{i k}} \leq 1, \forall k$.

Esse é um Problema de Programação Fracionária (PPF), resolvido para cada DMUs da amostra, onde $E f f_{o}$ (função objetivo) é a eficiência da DMU o em análise; $\mathrm{v}_{\mathrm{i}}$ e $\mathrm{u}_{\mathrm{j}}$ são os pesos de inputs $i, i=1, \ldots, r$, e outputs $j, j=1, \ldots, s$ respectivamente; $\mathrm{x}_{\mathrm{ik}}$ e $\mathrm{y}_{\mathrm{jk}}$ são os inputs $i$ e outputs $j$ da DMU $k, k=1, \ldots, n ; x_{i o} \mathrm{e}$ $y_{j o}$ são os inputs $i$ e outputs $j$ da DMU 0. A solução envolve a determinação dos valores dos pesos (também chamados de multiplicadores) para $v_{i}$ e $u_{j}$ (o peso de cada insumo i e produto j), a fim de que a medida de eficiência $\left(E f f_{o}\right)$ para a DMUo analisada seja maximizada, sujeita à restrição de que as medidas de eficiência de todas as unidades sejam menor ou igual a um. Desse modo, a eficiência relativa da DMUo analisada, definida como a soma ponderada dos produtos (Produto virtual), dividida pela soma ponderada dos insumos (Insumo virtual), devido à restrição do problema, sempre tomará valores entre 0 e 1 , sendo 1 (100\%) é considerada eficiente, e se 
menor que 1 ou igual a zero, será considerada ineficiente (MELLO et al., 2005; PEÑA, 2008; FERREIRA; GOMES, 2009).

O modelo CCR com orientação ao produto (output) ou ao insumo (input) pressupõe que as DMUs avaliadas operam com retornos constantes de escalas. Segundo Vasconcellos e Oliveira (1996) estudos empíricos mostram que a maior parte dos setores produtivos se encontra otimizando sua produção com retornos constantes de escalas. Entretanto, em situações de concorrência imperfeita, principalmente no setor público, existem organizações que podem estar operando com retornos crescentes e decrescentes de escalas, ou seja, com o modelo denominado de BCC, que considera retornos variáveis de escala (PEÑA, 2008).

Posteriormente ao modelo CCR da DEA, foi estendido por Banker, Charnes e Cooper (1984) para incluir retornos variáveis de escala (VRS - Variable Returns to Scale), ou seja, substitui o axioma da proporcionalidade entre inputs e outputs pelo axioma da convexidade, e que passou a ser chamado de BCC, devido as inicias dos nomes dos autores. Assim, os modelos básicos de DEA são conhecidos como CCR (ou CRS) e BCC (ou VRS). O modelo BCC também pode ser analisado sob duas formas de maximizar a eficiência, através da orientação ao insumo ou orientação ao produto (PEÑA, 2008).

Nos últimos anos, o método DEA vem sendo desenvolvido por diversos pesquisadores em todo o mundo, e aumentado sua aceitação principalmente por se tratar de um método totalmente objetivo, sem precisar necessariamente para determinação do modelo, a expertise do pesquisador (SENRA et. al., 2007).

\subsection{Modelo de Regressão Tobit}

Em muitas análises estatísticas de dados individuais, a variável dependente pode ser censurada ou truncada, ou seja, os dados variam dentro de um determinado intervalo (GREENE, 2008). Se a variável dependente é censurada para uma fração significativa das observações, as estimativas obtidas dos parâmetros pelo método de regressão linear são tendenciosas. Em 1958, James Tobin propôs um modelo que estimava os parâmetros, levando em consideração a existência das variáveis censuradas, tornando as estimativas consistentes (TOBIN, 1958). Pelas semelhanças que apresentava com as formulações dos modelos de regressão Probit e Logit, o modelo ficou sendo conhecido por Tobit (MADDALA, 1983).

No modelo de regressão Tobit (HENNINGSEN, 2010), temos uma variável dependente $y$ que é censurada à esquerda no zero, que pode ser visto nas seguintes expressões:

$y_{i}^{*}=x_{i}^{\prime} \beta+\varepsilon_{i}$

$y_{i}=\left\{\begin{array}{l}0, \text { se } y_{i}^{*} \leq 0 \\ y_{i}^{*}, \text { se } y_{i}^{*}>0\end{array}\right.$

onde o índice $\mathrm{i}=1, \ldots, \mathrm{N}$ indica as observações, $\mathrm{y}^{\star} \mathrm{e}$ uma variável não observável "latente", xi é um vetor de variáveis explicativas, $\beta$ é um vetor de parâmetros desconhecidos e ci é o erro.

A generalização do modelo de regressão Tobit é o Modelo de Regressão Censurada (HENNINGSEN, 2010). A variável dependente pode ser censurada à esquerda, censurada à direita e pode ser censurada à esquerda e à direita ao mesmo tempo, onde o limite inferior ou superior da variável dependente pode ser qualquer número:

$y_{i}^{*}=x_{i}^{\prime} \beta+\varepsilon_{i}$

$y_{i}=\left\{\begin{array}{l}a, \text { se } y_{i}^{*} \leq a \\ y_{i}^{*}, \text { se } a<y_{i}^{*}<b \\ b, \text { se } y_{i}^{*} \geq b\end{array}\right.$

Nessa expressão (5), $a$ é o limite inferior e $b$ é o limite superior da variável dependente. Se $a=-\infty$ ou $b=\infty$, a variável dependente não é censurada à esquerda nem censurada à direita.

O modelo de regressão Tobit foi selecionado para o estudo por sua presença em vários casos na literatura (ÇELEN, 2013) nas análises em segundo estágio e por ser considerado adequado para as análises com escores censurados. Segundo alguns autores (RAY, 1988; JI; LEE, 2010), o primeiro estágio seriam os escores de eficiência obtidos por meio do método DEA. 


\section{PROCEDIMENTOS E MÉTODOS}

Para atingir o objetivo proposto neste artigo, os procedimentos e métodos propostos foram as pesquisas bibliográficas, pesquisas documentais, a definição de variáveis e os métodos paramétrico e não paramétrico de análise dos dados.

\subsection{Amostra Pesquisada}

Adotou-se uma amostra de 63 Universidades Federais, pois corresponde ao total das universidades federais existentes no país, de uma população de 106 IFES até 2013 (INEP, 2013). Como a pesquisa está sendo realizada no âmbito de uma universidade (UnB), então para efeitos comparativos do estudo, optou-se por ter uma amostra com todas as universidades federais. No anexo A consta a relação completa das 63 universidades federais localizadas no país em 2013.

\subsection{Definição das Variáveis}

Existem muitas variáveis que podem ser utilizadas nos estudos de eficiência na área de educação com base no método DEA, porque envolvem grandes áreas de atividades de ensino, extensão e pesquisa (PEÑA, 2008), mas há limitações de disponibilidade dessas informações. De acordo com Costa, Ramos e Souza (2010), os outputs (produtos) educacionais são os resultados dos serviços prestados pelas IFES e os inputs (insumos) educacionais são os recursos que geram os serviços oferecidos pelas IFES. A questão fundamental está na definição das variáveis de entrada (input) e saída (output). Nos trabalhos sobre a eficiência das universidades públicas federais, foram considerados por Costa et. al. (2012), Oliveira (2013) e Siqueira (2015), os indicadores do TCU de números 1, 2, 3, 4, 5, 6 e 7 como sendo variáveis de Inputs, e os indicadores 8 e 9 como sendo variáveis de Outputs, conforme apresentado no Quadro 1.

\subsection{Aspectos Metodológicos}

No estudo da eficiência, utilizou-se para os cálculos do grau de eficiência das Unidades Tomadoras de Decisão (as Universidades Federais) o software DEAP (versão 2.1). O Software, desenvolvido por
Quadro 1 Variáveis de tipo Input e Output

\begin{tabular}{l|c}
\hline \multicolumn{1}{c|}{ Indicador } & $\begin{array}{c}\text { Tipo de } \\
\text { Variável }\end{array}$ \\
\hline 1. Custo corrente / Aluno equivalente & (Input) \\
\hline 2. Aluno tempo integral / Professor & (Input) \\
\hline 3. Aluno tempo integral / Funcionário & (Input) \\
\hline 4. Funcionário / Professor & (Input) \\
\hline 5. Grau de participação estudantil (GPE) & (Input) \\
\hline 6. Grau de envolvimento com pós-graduação (GEPG) & (Input) \\
\hline 7. Índice de qualificação do corpo docente (IQCD) & (Input) \\
\hline 8. Conceito CAPES/MEC para pós-graduação & (Output) \\
\hline 9. Taxa de sucesso na graduação (TSG) & (Output) \\
\hline
\end{tabular}

Fonte Elaboração própria, com base na Pesquisa Bibliográfica 2016.

Coelli (1996), permite mensurar por meio de inputs e outputs já definidos, calcular os ganhos de eficiência das IFES.

A DEA é um método que é usado para avaliar a eficiência técnica relativa individual em relação a um conjunto de Unidades Tomadoras de Decisão (DMU - Decision Making Unit), com múltiplas variáveis de inputs (insumos) e múltiplas de outputs (FERREIRA; GOMES, 2009). A definição de primeiro estágio, utilizada no método não paramétrico da DEA, são os valores calculados para os escores de eficiência técnica das IFES.

A definição de segundo estágio, na análise de eficiência DEA, é feita utilizando o modelo de regressão Tobit que é uma técnica de análise de regressão utilizada quando os valores da variável dependentes são censurados ou truncados em certos valores (HENNINGSEN, 2010). O método Tobit é considerado adequado, devido aos valores limites dos escores de eficiência obtidos através da DEA (JI; LEE, 2010) variarem entre 0 e 1 . No segundo estágio, o software utilizado para análise do modelo paramétrico de regressão Tobit foi o Stata 13.0.

No modelo de regressão Tobit, a variável depende (y) neste estudo são os escores de eficiência calculados pelo método DEA. As variáveis independentes ou explicativas $\left(\mathrm{x}_{\mathrm{i}}\right)$ são chamadas de variáveis ambientais. As variáveis ambientais foram escolhidas por não estarem diretamente relacionadas às variáveis usadas como insumos e produtos na análise DEA no 
primeiro estágio. Outros autores, como Ray (1988), Çelen (2013) e Fernandes e Resende Filho (2017), também consideram que as características ambientais não devem ter relação com os fatores de produção das DMUs. As variáveis ambientais deste estudo foram selecionadas por descreverem as situações envolvendo as áreas (Trabalho e Rendimento, Educação, Economia, Saúde, e Território e Ambiente) que impactam as IFES nos municípios onde elas estão localizadas, mas não tem relação direta com os insumos e os produtos utilizados no primeiro estágio. Segue na Tabela 1, as variáveis ambientais selecionadas para essa pesquisa.

\section{ANÁLISE DOS RESULTADOS}

A análise dos resultados será apresentada em duas partes: a primeira sendo a análise de eficiência calculada em 2015 e a segunda sendo a análise de regressão Tobit para validação do modelo de eficiência.

\section{PARTE 1: Análise de eficiência em 2015}

No ano de 2015 foi feita a análise de eficiência utilizando-se um modelo DEA com retornos constantes de escala (CCR), com orientação a output.
Observa-se na Tabela 2 a saída do resultado feito pelo software DEAP, com os cálculos das eficiências técnicas para cada universidade. Os resultados apresentam as IFES que estão na fronteira de eficiência, ou seja, são as que possuem eficiência técnica igual a 1,0. As IFES ineficientes também aparecem nessa tabela com eficiência técnica menor que 1,0 .

Em 2015, na Tabela 2 foram encontradas 24 IFES consideradas eficientes, representando $44 \%$ das IFES que estão na fronteira de eficiência. Sendo que seis dessas IFES serviram de referência (benchmark) por mais de dez vezes para as outras IFES ineficientes. Veja a lista completa de referências no Anexo B. As seis IFES são, por ordem decrescente de quantidades de vezes que serviram de referências, a Universidade Federal do Estado do Rio de Janeiro - UNIRIO (26 vezes), a Universidade Federal de Minas Gerais UFMG (15 vezes), a Universidade Federal dos Vales do Jequitinhonha e Mucuri - UFVJM (15 vezes), a Universidade Federal do Amapá - UNIFAP (14 vezes), a Universidade Federal do Pará - UFPA (12 vezes) e a Universidade Federal do Rio de Janeiro - UFRJ (11 vezes). Isso mostra o grau de importância dessas IFES em relação às que são eficientes.

Quanto às 30 IFES que não são eficientes e, que representam $56 \%$ da amostra, podemos destacar as

Tabela 1 Variáveis Ambientais

\begin{tabular}{|c|c|c|}
\hline Áreas & Código da Variável & Nome das Variáveis Ambientais \\
\hline Trabalho e Rendimento & TR2 & Pessoal ocupado [2015] \\
\hline Trabalho e Rendimento & TR3 & Percentual da População ocupada [2015] \\
\hline Educação & ED1 & Taxa de escolarização de 6 a 14 anos de idade [2010] \\
\hline Educação & ED4 & Matrículas no ensino fundamental [2015] \\
\hline Educação & ED5 & Matrículas no ensino médio [2015] \\
\hline Educação & ED6 & Docentes no ensino fundamental [2015] \\
\hline Educação & ED7 & Docentes no ensino médio [2015] \\
\hline Educação & ED10 & Matriculas/Docentes do ensino Fundamental \\
\hline Educação & ED11 & Matriculas/Docentes do ensino Médio \\
\hline Economia & EC1 & PIB per capita [2015] \\
\hline Saúde & SA2 & Internações por diarreia [2016] \\
\hline Território e Ambiente & TA3 & Arborização de vias públicas [2010] \\
\hline
\end{tabular}

Fonte: Elaboração própria, com base no sistema Brasil em Síntese no site do IBGE (2017). 
Tabela 2 Resultados da Eficiência Técnica das IFES em 2015

\begin{tabular}{|c|c|c|}
\hline Código & SIGLA das IFES & Eficiência Técnica \\
\hline 1 & UFPR & 1,000 \\
\hline 2 & UFRJ & 1,000 \\
\hline 3 & UFV & 1,000 \\
\hline 4 & UFMG & 1,000 \\
\hline 5 & UFRGS & 1,000 \\
\hline 6 & UFC & 1,000 \\
\hline 7 & UFPA & 1,000 \\
\hline 8 & UFF & 1,000 \\
\hline 9 & UFSC & 1,000 \\
\hline 10 & UFAL & 1,000 \\
\hline 11 & UFPEL & 1,000 \\
\hline 12 & UFAC & 1,000 \\
\hline 13 & UNIRIO & 1,000 \\
\hline 14 & UFSJ & 1,000 \\
\hline 15 & UNIFAP & 1,000 \\
\hline 16 & UFLA & 1,000 \\
\hline 17 & UNIFESP & 1,000 \\
\hline 18 & UFRA & 1,000 \\
\hline 19 & UFABC & 1,000 \\
\hline 20 & UFVJM & 1,000 \\
\hline 21 & UFCSPA & 1,000 \\
\hline 22 & UNIPAMPA & 1,000 \\
\hline 23 & UFCA & 1,000 \\
\hline 24 & UNIFESSPA & 1,000 \\
\hline 25 & UFTM & 0,996 \\
\hline 26 & UNIFAL & 0,989 \\
\hline 27 & UFU & 0,985 \\
\hline 28 & UFPI & 0,979 \\
\hline 29 & UFRR & 0,975 \\
\hline 30 & UNIFEI & 0,959 \\
\hline 31 & UFMA & 0,953 \\
\hline 32 & UFBA & 0,950 \\
\hline 33 & UFOP & 0,925 \\
\hline 34 & UFG & 0,923 \\
\hline 35 & UFSCar & 0,923 \\
\hline 36 & UFJF & 0,915 \\
\hline 37 & UFPE & 0,905 \\
\hline 38 & UnB & 0,903 \\
\hline 39 & UFERSA & 0,892 \\
\hline 40 & UFAM & 0,889 \\
\hline 41 & UNIR & 0,887 \\
\hline 42 & UFRRJ & 0,886 \\
\hline 43 & UFRN & 0,883 \\
\hline 44 & UFES & 0,872 \\
\hline 45 & UFCG & 0,868 \\
\hline 46 & UFSM & 0,866 \\
\hline 47 & UFRPE & 0,859 \\
\hline 48 & UFFS & 0,856 \\
\hline 49 & UNIVASF & 0,851 \\
\hline 50 & UFGD & 0,850 \\
\hline 51 & UFMT & 0,840 \\
\hline 52 & UFPB & 0,835 \\
\hline 53 & UFMS & 0,794 \\
\hline \multirow[t]{2}{*}{54} & FURG & 0,769 \\
\hline & Média & 0,944 \\
\hline
\end{tabular}

Fonte: Elaboração própria, com base na saída do software DEAP 2.1. seis mais ineficientes (valores de eficiência abaixo de um) que são a Universidade Federal do Vale do São Francisco - UNIVASF (0,851), a Universidade Federal da Grande Dourados - UFGD $(0,850)$, a Universidade Federal de Mato Grosso - UFMT $(0,840)$, a Universidade Federal da Paraíba - UFPB $(0,835)$, Universidade Federal de Mato Grosso do Sul - UFMS $(0,794)$ e a Universidade Federal do Rio Grande - FURG (0,769).

No ano de 2015, para o conjunto das 54 IFES houve uma redução, em média, da eficiência na ordem de 5,6\%, ou seja, a média do nível de eficiência em 2015 foi de 0,944 .

\section{PARTE 2: Validação do modelo de Eficiência}

A validação do modelo de eficiência é verificada por meio da análise de regressão Tobit, para o segundo estágio do método DEA, com os dados censurados a direita, cujos resultados são apresentados no Quadro 2.

No Quadro 2, observa-se os resultados apresentados na coluna do teste dos coeficientes (P) das variáveis ambientais, e considerando um nível de significância de $5 \%(0,05)$ para testar a hipótese de nulidade dos coeficientes, ou seja, valores de teste menores ou iguais a 0,05 rejeitaria a hipótese de nulidade do coeficiente, tornando significativas a variáveis no modelo.

Considerando os valores encontrados na coluna do teste dos coeficientes $(\mathrm{P})$, as variáveis ambientais TR2 (Pessoal ocupado), ED7 (Docentes no ensino médio) e ED11 (Matriculas/Docentes do ensino Médio) foram consideradas significativas para o modelo e apresentaram o sinal positivo como esperado. Também encontramos variáveis do modelo que tiveram o sinal negativo, mas foram rejeitadas pelo teste, ou seja, não são significativas no modelo. E outras variáveis que apesar de terem sinal positivo, mas que foram aceitas as hipóteses de nulidade dos seus coeficientes, que são as ED4 (Matrículas no ensino fundamental), EC1 (PIB per capita) e SA2 (Internações por diarreia), ou seja, também não são significativas no modelo.

Nota-se também que houve a validação do modelo utilizando os escores de eficiência da DEA, pois o valor encontrado no teste (Prob > chi2) de 
Quadro 2 Regressão sobre escores DEA

- tobit Y TR2 TR3 ED1 ED4 ED5 ED6 ED7 ED10 ED11 EC1 SA2 TA3, ul (10) Tobit regression Number of obs = 54 LR $\operatorname{chi2}(12) \quad=$

\begin{tabular}{r|rrrrrr}
\hline Y & Coef. & Std. Err. & $t$ & $\mathrm{P}>|\mathrm{t}|$ & {$[958$ Conf. Interval] } \\
\hline TR2 & $4.43 e-06$ & $1.95 e-06$ & 2.28 & 0.028 & $5.02 e-07$ & $8.36 \mathrm{e}-06$ \\
TR3 & -.0359247 & .0195747 & -1.84 & 0.074 & -.0754279 & .0035786 \\
ED1 & -.1598707 & .182327 & -0.88 & 0.386 & -.5278214 & .2080801 \\
ED4 & .0000115 & .0000206 & 0.56 & 0.581 & -.0000302 & .0000531 \\
ED5 & -.0000893 & .0000592 & -1.51 & 0.139 & -.0002089 & .0000302 \\
ED6 & -.0005158 & .0004098 & -1.26 & 0.215 & -.0013428 & .0003111 \\
ED7 & .001418 & .0007186 & 1.97 & 0.055 & -.0000322 & .0028682 \\
ED10 & -.2128663 & .1191184 & -1.79 & 0.081 & -.4532569 & .0275242 \\
ED11 & .2508167 & .1192061 & 2.10 & 0.041 & .010249 & .4913844 \\
EC1 & $1.87 e-06$ & .0000173 & 0.11 & 0.914 & -.000033 & .0000368 \\
SA2 & .5259733 & .3050842 & 1.72 & 0.092 & -.0897115 & 1.141658 \\
TA3 & -.008222 & .0060328 & -1.36 & 0.180 & -.0203968 & .0039527 \\
Cons & 26.92117 & 18.53177 & 1.45 & 0.154 & -10.47747 & 64.3198 \\
\hline /sigma & .8515746 & .1196137 & & & .6101845 & 1.092965 \\
\hline
\end{tabular}

obs . summary:

$\begin{array}{rr}0 & \text { left-censored observations } \\ 30 & \text { uncensored observations } \\ 24 & \text { right-censored observations at } Y>=10\end{array}$

Fonte: Elaboração própria, com base na saída do software Stata 13.0.

ajuste do modelo foi de 0,0378 , menor que o valor estabelecido para testar a validade do modelo, que é um nível de significância menor ou igual 5\% $(0,05)$. Sendo assim, verificamos que o modelo ajustado pode ser representado pela relação da magnitude do efeito das variáveis ambientais sobre os escores de eficiência.

\section{CONSIDERAÇÕES FINAIS}

Nesta pesquisa buscou-se avaliar o desempenho das IFES, quanto à eficiência em relação ao custo-benefício dos gastos de recursos públicos do programa REUNI em 2015 e verificar a validação dessa eficiência das IFES.

Implicitamente, para o governo, era de se esperar que houvessem ganhos de eficiência para as universidades públicas federais brasileiras devido à injeção de recursos públicos nas IFES, porém o que se observou em 2015 foi que menos da metade (44\%) das IFES são consideradas eficientes. E dentre essas destacam-se a Universidade Federal do Estado do Rio de Janeiro, a Universidade Federal de Minas Gerais, a Universidade Federal dos Vales do Jequitinhonha e Mucuri, a Universidade Federal do Amapá, a Universidade Federal do Pará e a Universidade Federal do Rio de Janeiro.

Apesar de termos $44 \%$ das IFES eficientes, a média do nível de eficiência em 2015 foi de 0,944, isso pode indicar um bom desempenho do nível de eficiência, pois $70 \%$ das IFES possuem um coeficiente acima de 0,90 (90\%), significando que está mais próximo de $1(100 \%)$. 
Foram observadas $56 \%$ das IFES como ineficientes, sendo as que se destacaram com os piores desempenhos a Universidade Federal do Vale do São Francisco, a Universidade Federal da Grande Dourados, a Universidade Federal de Mato Grosso, a Universidade Federal da Paraíba, a Universidade Federal de Mato Grosso do Sul e a Universidade Federal do Rio Grande.

De acordo com os resultados da presente pesquisa, verificou-se que houve a validação do modelo ajustado que considerava a relação das IFES eficientes e não eficientes, utilizando os insumos e produtos estabelecidos, com as variáveis ambientais selecionadas, pois o teste de ajuste do modelo de regressão Tobit foi considerado significativo (0.0378). E as variáveis que foram consideradas significativas para o ajuste do modelo foram as "Pessoal ocupado", "Docentes no ensino médio" e "Matriculas/Docentes do ensino Médio".

Sugere-se que pesquisas futuras utilizem novas variáveis de insumos e produtos, assim como outras variáveis ambientais que possam corroborar com os testes aplicados nesse estudo. Por fim, também seria cabível para complementar as pesquisas envolvendo esse tema, análises em segundo estágio utilizando outras metodologias para as regressões, conforme as propostas por Simar e Wilson (2007) e Banker e Natarajan (2008).

\section{REFERÊNCIAS}

BANKER, R. D.; CHARNES, A.; COOPER, W.W. Some models for estimating technical and scale inefficiencies in Data Envelopment Analysis. Management Science, v.30, n. 9, 1078-1092, 1984.

BANKER, R. D.; NATARAJAN, R. Evaluating contextual variables affecting productivity using data envelopment analysis. Operations research, INFORMS, v. 56, n. 1, p. $48-58,2008$.

BARBOSA, G. C.; FREIRE, F. S.; CRISÓSTOMO, V. L. Análise dos indicadores de gestão das IFES e o desempenho discente no ENADE. Revista da avaliação da educação superior, Campinas, v. 16, n. 2, p. 317-344, jul. 2011.
BELLONI, J. A. Uma Metodologia de Avaliação da Eficiência Produtiva de Universidades Federais Brasileiras. 245p. Tese de Doutorado. Universidade Federal de Santa Catarina, Florianópolis, 2000.

BRASIL. Constituição (1988). Constituição da República Federativa do Brasil. Disponível em: $<$ http://www.planalto.gov.br/ccivil_03/Constituicao/ Constituicao.htm>. Acesso em: 13 out. 2015.

Decreto $\mathrm{n}^{\circ}$ 6.096, de 24 de abril de 2007. Institui o Programa REUNI. Brasília, 2007. Em http:// www.planalto.gov.br/ccivil_03/_ato2007-2010/2007/ decreto/d6096.htm. Acesso 13 out 2015.

. Lei ${ }^{\circ} 10.172$, de 9 de janeiro de 2001. Aprova o Plano Nacional de Educação e dá outras providencias. Disponível em: <http://www.planalto.gov.br/ ccivil_03/leis/leis_2001/110172.htm>. Acesso em: 13 out. 2015.

. Ministério da Educação. Balanço Social 20032015. Brasília, 2016. 94 p.

. Tribunal de Contas da União - TCU; Secretaria de Educação Superior - SESu/MEC; Secretaria Federal de Controle Interno - SFC. Orientações para o cálculo dos indicadores de gestão: decisão TCU no 408/2002 - plenário. Versão revisada março 2004. In: Ministério da Educação. Disponível em: <http://portal.mec.gov.br/sesu/arquivos/pdf/ indicadores.pdf $>$. Acesso 13 out. 2015.

CAVALCANTE, S. M.; ANDRIOLA, W. Avaliação da Eficiência dos Cursos de Graduação da Universidade Federal do Ceará (UFC) através da Análise Envoltória de Dados (DEA). Revista Iberoamericana de Evaluación Educativa, v. 5 n. 3, p. 290-313, 2012.

ÇELEN, A. Efficiency and productivity (tfp) of the turkish electricity distribution companies: An application of two-stage (dea\&tobit) analysis. Energy Policy, Elsevier, v. 63, p. 300-310, 2013. 
CHARNES, A.; COOPER, W.W; RHODES, E. Measuring the efficiency of decision making units. European Journal of Operational Research, v.2, p.429444, 1978.

COELLI, T. J. A guide to DEAP version 2.1: a DEA (computer) program. Centre for Efficiency and Productivity Analysis (CEPA) WP 8, University of New England, Austrália, 1996.

COELLI, T. J.; RAO, D. S. P.; O’DONNELL, C. J.; BATTESE, G. E. An introduction to efficiency and productivity analysis. Springer Science \& Business Media, 2005.

COSTA, D, M.; PAIVA, R. V. C.; FERREIRA, J. C. P. A educação Superior tecnológica como um caminho para a expansão da educação superior no Brasil. In: X Colóquio Internacional sobre Gestão Universitária na América do Sul. Argentina, 2010.

COSTA, E. M.; RAMOS, F.; SOUSA, H. R. Mensuração de eficiência produtiva das Instituições Federais de Ensino Superior (IFES). Brasilia, DF, Esaf, 2010.

COSTA, E. M.; SOUZA, H. R.; RAMOS, F. S.; SILVA, J. L. M. Eficiência e desempenho no ensino superior: uma análise da fronteira de produção educacional das IFES brasileiras. Revista de Economia Contemporânea, v. 16, n. 3, p. 415-440, 2012.

FERNANDES, D. P.; RESENDE FILHO, M. D. A. Eficiência de custos operacionais das companhias de distribuição de energia elétrica (cdees) no brasil: Uma aplicação (DEA \& Tobit) em dois estágios. 2017.

FERREIRA, C. M. C; GOMES, A. P. Introdução à análise envoltória de dados: teoria, modelos e aplicações. Editora UFV, Viçosa, MG, 2009.

GREENE, W.H. Econometric Analysis. Prentice Hall, 6th edition, 2008.

HENNINGSEN, A.; Estimating censored regression models in $\mathrm{R}$ using the censReg Package - $\mathrm{R}$ package vignettes, 2010.
IBGE. Instituto Brasileiro de Geografia e Estatística. O Brasil em Síntese é o sistema agregador de informações do IBGE sobre os municípios e estados do Brasil. 2017. Disponível em < https://cidades.ibge. gov.br/> Acesso em 26 abr. 2018.

JI, Y.-B.; LEE, C. Data Envelopment Analysis. The Stata Journal, v. 10, n. 2, p. 267- 280, 2010.

MADDALA, G. S. Limited-dependent and qualitative variables in econometrics. Melbourne: Cambridge University Press, 1983.

MELLO, J. C. C. B.; MEZA, L. A.; GOMES, E. G.; NETO, L. B. Curso de Análise de Envoltória de Dados. Anais do SBPO-XXXVII, p. 2520-2540, 2005.

MENEZES, E. T.; SANTOS, T. H. IFES (Instituições Federais de Ensino Superior), Dicionário Interativo da Educação Brasileira - EducaBrasil. São Paulo, Editora Midiamix, 2002.

OLIVEIRA, A. J. Programa REUNI nas Instituições de Ensino Superior Federal (IFES) Brasileiras: um estudo da eficiência operacional por meio da análise envoltória de dados (DEA) no período de 2006 a 2012. 2013. 146 f. Dissertação (Mestrado em Ciências Contábeis) - Programa de Mestrado em Contabilidade, Universidade Federal do Paraná, Curitiba, 2013.

PEÑA, C. R. Um Modelo de Avaliaçăo da Eficięncia da Administraçăo Pública através do Método Análise Envoltória de Dados (DEA). Revista de Administração Contemporânea, Curitiba, Paraná, v. 12, n. 1, p. 83-106, 2008.

RIOS, L. R. Medindo a eficiência relativa das operações dos terminais de contêineres do MERCOSUL. Dissertação Mestrado, UFRGS, Porto Alegre, RS, 2005.

RAY, S. C. Data envelopment analysis, nondiscretionary inputs and efficiency: an alternative interpretation. Socio-Economic Planning Sciences, Elsevier, v. 22, n. 4, p. 167-176, 1988. 
SAVIANI, D. O Plano de Desenvolvimento da Educação: Análise do projeto do MEC, Revista Educação \& Sociedade, Campinas, vol. 28, n. 100 Especial, p. 1231-1255, out. 2007.

SENRA, L. F. A. C.; NANCI, L. C.; MELLO, J. C. C. B. S.; MEZA, L. A. Estudo sobre métodos de seleção de variáveis em DEA. Pesquisa Operacional, v. 27, n. 2, p. 191-207, 2007.

SILVA, C. T.; MORGAN, B. F.; CUNHA, J. R.; MOURA, J. D.; FILHO, J. R.; COSTA, P. D. Custos no Setor Publico. Brasília, Editora Universidade de Brasília, 2007.

SILVA, R. L.; FREITAS, F. C. H. P.; LINS, M. T. G. A implantação do programa de Reestruturação e Expansão das Universidades Federais/REUNI: um estudo de Caso. Revista Gestão Universitária na América Latina-GUAL, v. 6, n. 4, p. 147-170, 2013.

SIMAR, L.; WILSON, P. W. Estimation and inference in two-stage, semi-parametric models of production processes. Journal of econometrics, Elsevier, v. 136, n. 1, p. 1-64, 2007.

SIQUEIRA, J. S. Eficiência das universidades públicas federais brasileiras: Um estudo com foco no projeto REUNI. Dissertação de Mestrado, Universidade Federal de Campina Grande, PB, 2015.

TOBIN, J.; Estimation of relationship for limited dependent variables. Econometrica, v. 26, p. 24-36, 1958.

VASCONCELLOS, M. A. S.; OLIVEIRA, R. Manual de Microeconomia. São Paulo, Atlas, 1996. 


\section{ANEXOS}

\section{Anexo A: Relação das Universidades Federais Brasileiras em 2013}

\begin{tabular}{|c|c|c|c|c|c|}
\hline Seq. & Nome & Sigla & Seq. & Nome & Sigla \\
\hline 1 & Universidade de Brasília & UnB & 33 & Univ. Federal do Sul e Sudeste do Pará & UNIFESSPA \\
\hline 2 & Universidade Federal da Grande Dourados & UFGD & 34 & Universidade Federal de Alfenas & UNIFAL \\
\hline 3 & Universidade Federal de Goiás & UFG & 35 & Universidade Federal de Itajubá & UNIFEI \\
\hline 4 & Universidade Federal de Mato Grosso & UFMT & 36 & Universidade Federal de Juiz de Fora & UFJF \\
\hline 5 & Universidade Federal de Mato Grosso do Sul & UFMS & 37 & Universidade Federal de Lavras & UFLA \\
\hline 6 & Universidade Federal da Bahia & UFBA & 38 & Universidade Federal de Minas Gerais & UFMG \\
\hline 7 & Universidade Federal do Sul da Bahia & UFSB & 39 & Universidade Federal de Ouro Preto & UFOP \\
\hline 8 & Universidade Federal do Recôncavo da Bahia & UFRB & 40 & Universidade Federal de São Carlos & UFSCar \\
\hline 9 & $\begin{array}{l}\text { Universidade Federal da Integração } \\
\text { Internacional da Lusofonia Afro-Brasileira }\end{array}$ & UNILAB & 41 & Universidade Federal de São João del-Rei & UFSJ \\
\hline 10 & Universidade Federal da Paraíba & UFPB & 42 & Universidade Federal de São Paulo & UNIFESP \\
\hline 11 & Universidade Federal do Cariri & UFCA & 43 & Universidade Federal de Uberlândia & UFU \\
\hline 12 & Universidade Federal de Alagoas & UFAL & 44 & Universidade Federal de Viçosa & UFV \\
\hline 13 & Universidade Federal de Campina Grande & UFCG & 45 & Universidade Federal do ABC & UFAB \\
\hline 14 & Universidade Federal de Pernambuco & UFPE & 46 & Universidade Federal do Espírito Santo & UFES \\
\hline 15 & Universidade Federal de Sergipe & UFS & 47 & Univ. Federal do Estado do Rio de Janeiro & UNIRIO \\
\hline 16 & Universidade Federal do Ceará & UFC & 48 & Universidade Federal do Rio de Janeiro & UFRJ \\
\hline 17 & Universidade Federal do Maranhão & UFMA & 49 & Universidade Federal do Triângulo Mineiro & UFTM \\
\hline 18 & Universidade Federal do Oeste da Bahia & UFOB & 50 & $\begin{array}{l}\text { Universidade Federal dos Vales } \\
\text { do Jequitinhonha e Mucuri }\end{array}$ & UFVJM \\
\hline 19 & Universidade Federal do Piauí & UFPI & 51 & Universidade Federal Fluminense & UFF \\
\hline 20 & Univ. Federal do Rio Grande do Norte & UFRN & 52 & Universidade Federal Rural do Rio de Janeiro & UFRRJ \\
\hline 21 & $\begin{array}{l}\text { Universidade Federal do } \\
\text { Vale do São Francisco }\end{array}$ & UNIVASF & 53 & Universidade Federal da Fronteira Sul & UFFS \\
\hline 22 & Universidade Federal Rural de Pernambuco & UFRPE & 54 & Univ. Fed. da Integração Latino-Americana & UNILA \\
\hline 23 & Universidade Federal Rural do Semi-Árido & UFERSA & 55 & $\begin{array}{l}\text { Universidade Federal de Ciências } \\
\text { da Saúde de Porto Alegre }\end{array}$ & UFCSPA \\
\hline 24 & Universidade Federal de Rondônia & UNIR & 56 & Universidade Federal de Pelotas & UFPEL \\
\hline 25 & Universidade Federal de Roraima & UFRR & 57 & Universidade Federal de Santa Catarina & UFSC \\
\hline 26 & Universidade Federal do Acre & UFAC & 58 & Universidade Federal de Santa Maria & UFSM \\
\hline 27 & Universidade Federal do Amapá & UNIFAP & 59 & Universidade Federal do Pampa & UNIPAMPA \\
\hline 28 & Universidade Federal do Amazonas & UFAM & 60 & Universidade Federal do Paraná & UFPR \\
\hline 29 & Universidade Federal do Oeste do Pará & UFOPA & 61 & Universidade Federal do Rio Grande & FURG \\
\hline 30 & Universidade Federal do Pará & UFPA & 62 & Universidade Federal do Rio Grande do Sul & UFRGS \\
\hline 31 & Universidade Federal do Tocantins & UFT & 63 & Universidade Tecnológica Federal do Paraná & UTFPR \\
\hline 32 & Universidade Federal Rural da Amazônia & UFRA & & & \\
\hline
\end{tabular}

Fonte: Elaboração própria, com base na Pesquisa Bibliográfica 2016. 
Anexo B: Dados das IFES de referência (benchmarks) no ano de 2015

\begin{tabular}{|c|c|c|}
\hline Código & SIGLA das IFES & $\begin{array}{c}\text { Referência } \\
\text { (no de vezes) }\end{array}$ \\
\hline 1 & FURG & 0 \\
\hline 2 & UFABC & 8 \\
\hline 3 & UFAC & 0 \\
\hline 4 & UFAL & 10 \\
\hline 5 & UFAM & 0 \\
\hline 6 & UFBA & 0 \\
\hline 7 & UFC & 0 \\
\hline 8 & UFCA & 6 \\
\hline 9 & UFCG & 0 \\
\hline 10 & UFCSPA & 3 \\
\hline 11 & UFERSA & 0 \\
\hline 12 & UFES & 0 \\
\hline 13 & UFF & 0 \\
\hline 14 & UFFS & 0 \\
\hline 15 & UFG & 0 \\
\hline 16 & UFGD & 0 \\
\hline 17 & UFJF & 0 \\
\hline 18 & UFLA & 9 \\
\hline 19 & UFMA & 0 \\
\hline 20 & UFMG & 15 \\
\hline 21 & UFMS & 0 \\
\hline 22 & UFMT & 0 \\
\hline 23 & UFOP & 0 \\
\hline 24 & UFPA & 12 \\
\hline 25 & UFPB & 0 \\
\hline 26 & UFPE & 0 \\
\hline 27 & UFPEL & 9 \\
\hline
\end{tabular}

\begin{tabular}{|c|c|c|}
\hline Código & SIGLA das IFES & $\begin{array}{c}\text { Referência } \\
\text { ( } n \text { ㅇ de vezes) }\end{array}$ \\
\hline 28 & UFPI & 0 \\
\hline 29 & UFPR & 0 \\
\hline 30 & UFRA & 3 \\
\hline 31 & UFRGS & 3 \\
\hline 32 & UFRJ & 11 \\
\hline 33 & UFRN & 0 \\
\hline 34 & UFRPE & 0 \\
\hline 35 & UFRR & 0 \\
\hline 36 & UFRRJ & 0 \\
\hline 37 & UFSC & 3 \\
\hline 38 & UFSCar & 0 \\
\hline 39 & UFSJ & 3 \\
\hline 40 & UFSM & 0 \\
\hline 41 & UFTM & 0 \\
\hline 42 & UFU & 0 \\
\hline 43 & UFV & 2 \\
\hline 44 & UFVJM & 15 \\
\hline 45 & UnB & 0 \\
\hline 46 & UNIFAL & 0 \\
\hline 47 & UNIFAP & 14 \\
\hline 48 & UNIFEI & 0 \\
\hline 49 & UNIFESP & 0 \\
\hline 50 & UNIFESSPA & 0 \\
\hline 51 & UNIPAMPA & 2 \\
\hline 52 & UNIR & 0 \\
\hline 53 & UNIRIO & 26 \\
\hline 54 & UNIVASF & 0 \\
\hline
\end{tabular}

Fonte: Saída do software DEAP 2.1 com adaptações. 